Relations industrielles

Industrial Relations

\title{
Murray, Gregor, Marie-Laure Morin et Isabel Da Costa, dir., L'État des relations professionnelles : traditions et perspectives de la recherche
}

\section{Dimitri Weiss}

Volume 52, numéro 3, 1997

URI : https://id.erudit.org/iderudit/051191ar

DOI : https://doi.org/10.7202/051191ar

Aller au sommaire du numéro

\section{Éditeur(s)}

Département des relations industrielles de l'Université Laval

ISSN

0034-379X (imprimé)

1703-8138 (numérique)

Découvrir la revue

Citer ce compte rendu

Weiss, D. (1997). Compte rendu de [Murray, Gregor, Marie-Laure Morin et Isabel Da Costa, dir., L'État des relations professionnelles : traditions et perspectives de la recherche]. Relations industrielles / Industrial Relations, 52(3), 654-655. https://doi.org/10.7202/051191ar

Tous droits réservés (C) Département des relations industrielles de l'Université Laval, 1997
Ce document est protégé par la loi sur le droit d'auteur. L'utilisation des services d'Érudit (y compris la reproduction) est assujettie à sa politique d'utilisation que vous pouvez consulter en ligne.

https://apropos.erudit.org/fr/usagers/politique-dutilisation/ 


\section{Recensions}

\section{Book Reviews}

\section{L'État des relations professionnelles: traditions et perspectives de la recherche \\ sous la direction de Gregor MURRAY, Marie-Laure MORIN, Isabel Da COSTA, Québec: Les Presses de l'Université Laval, Toulouse: Octares Éditions, 1996, 620 p., ISBN 2-7637-7483-0.}

Je me dois de dire, dès l'abord, qu'il s'agit d'un livre de prix, remarquablement conçu, agencé et coordonné, cultivant la diversité des approches et des points de vue, appellant, pour une part, des vues pertinemment comparées, et réunissant une brochette de chercheurs québécois et français égaux à eux-mêmes dans un effort de synthèse et de quête de l'essentiel. Il en est résulté un document de référence, destiné à éclairer, des deux côtés de l'Atlantique, la voie de ceux de leurs congénères concernés, à un titre ou un autre, par le vaste champ des relations industrielles.

Point de nouveaux termes ou d'excès de métaphores dans des textes qui, pour la plupart, unissent le sérieux de l'analyse à un effort d'information, fût-il synthétique, qui permet au lecteur de parcourir les transformations qui ont graduellement jalonné l'évolution du domaine. Ouvrage à domainante sociologique, mais dont ne sont pas absents les économistes et les juristes, voire, incidemment, des gestionnaires, mais qui - nous sommes en Amérique du Nord - peuvent, pour certains, se définir, à titre principal, comme des "relationnistes".

Le livre tire son origine d'un colloque organisé à l'Université Laval deux années auparavant, ce qui en explique la structure: une quarantaine d'interventions exposants et discutants - réparties en cinq parties : la constitution d'un champ d'études, entreprise et gestion du travail, mode de représentation et négociation collective, règles et marchés, transformations des relations professionnelles et perspectives de recherche. Le tout précédé d'un avant-propos et d'une présentation générale sous le titre, juste, "Regards croisés sur la recherche en relations professionnelles $"$.

En ajoutant que chaque partie commence par une présentation de très bonne qualité, on peut comprendre l'embarras du critique: tout est clairement résumé et, grâce aux discutants, objet de répliques autorisées. Faudrait-il alors que l'auteur du compte-rendu (re)présente les présentations et commente les commentaires? Il lui reste à (re)dire la place qu'à ses yeux, "L'état des relations professionnelles " occupe désormais dans la littérature spécialisée - la première - et dire sa satisfaction d'avoir vu, enfin, une telle initiative se concrétiser en un ensemble cohérent d'apports qui, en ce monde de l'immédiat et du court terme, s'inscrit dans la durée - ou, tout au moins, dans une certaine durée.

Ceux qui doutent toujours de l'intérêt des relations industrielles, - encore récemment définies dans un ouvrage italien spécialisé comme "l'ensemble des rapports entre employeurs et travailleurs qui s'expriment sous une forme contractuelle, plus ou moins intentionnelle, explicite, formalisée, en un quelconque environnement de travail ", vont trouver dans le volume franco-québécois de quoi 
nourrir un regard régénéré. Les faiblesses du champ ne sont pas tues, ni ses écueils et les aspects maturs, voire déclinants, qui entraient dans sa composition.

Le livre s'achève sur un constat encourageant: " les relations industrielles ont encore un rôle important à jouer dans la gestion des rapports humains". Acceptons-en l'augure!
Ses directeurs ont eu suffisamment de mérites pour leur reprocher, pêché véniel s'il en est, de n'avoir poussé l'effort jusqu'à l'ajout d'un index détaillé. Chaque lecteur attentif se verra, ainsi contraint, pour son plus grand bien, à le faire lui-même. Sain exercice.

DIMITRI WEISS

Université de Paris I - IAE

\section{Team Toyota: Transplanting the Toyota Culture to the Camry Plant in Kentucky}

par Terry L. BESSER, New York : State University of New York Press, 1996, 199 p., ISBN 0-7914-3145-2 et 0-7914-3146-0.

Les dirigeants de l'entreprise Toyota croient fermement que le succès de leur entreprise est dû, en grande partie, à la façon "Toyota" d'organiser le travail. Contrairement à la majorité des entreprises japonaises établies à l'étranger, Toyota a décidé d'exporter une version modifiée de son mode de gestion qui est à la base de la motivation des travailleurs tant japonais que non japonais. Dans son livre Team Toyota, Besser tente d'évaluer dans quelle mesure Toyota a réussi à transplanter, en tout ou en partie, ce mode de gestion dans sa nouvelle usine non syndiquée de production des Camrys au Kentucky (États-Unis). En particulier, l'auteur cherche à savoir si les travailleurs américains à l'instar des employés nippons ont réagi favorablement à ce mode de gestion à la japonaise.

Le sujet de recherche est très intéressant, car il peut ouvrir la voie à de nouvelles façons, peut-être plus efficaces, de gérer les ressources humaines. Traditionnellement, le mode de gestion privilégié aux États-Unis dans ce secteur industriel est de type bureaucratique. Estil possible que le modèle américain ne soit pas le meilleur (one best way) ou l'unique façon de gérer efficacement? Si cette question peut déranger certains dirigeants conservateurs, elle est très rafraîchissante intellectuellement. Pour étudier ce sujet, Besser a réalisé une étude de cas en utilisant une méthodologie qualitative (documents, entrevues, observation, etc.). Après avoir expliqué le sujet de recherche (chapitre 1), la méthodologie (chapitre 2) et le contexte (chapitre 3 ), l'auteur présente les résultats de son étude (chapitres 4 à 8 ). Plutôt que de décrire le contenu de chacun de ces chapitres, nous allons présenter les traits saillants des résultats.

Globalement, Toyota a réussi à garder au moins trois éléments de son mode de gestion: le concept d'équipe, l'idée d'amélioration continue, la notion de contribution au succès organisationnel. Premièrement, le concept d'équipe est central au mode de gestion à la Toyota tant au Japon qu'aux États-Unis. Les employés sont regroupés en petits groupes de quatre ou cinq personnes (team members) dont le travail est coordonné par l'un d'entre eux (team leader). Pour arriver à former des équipes de travail cohésives, Toyota a mis en place un système cohérent de pratiques de gestion des ressources humaines. Contrairement au modèle bureaucratique, les employés sont sélectionnés moins pour leur expertise technique (la plupart d'entre eux n'ayant pas ou peu d'expérience manufacturière) que pour leur capacité de travailler ensemble, leur loyauté et leur engagement (leur inexpérience aidant à modeler des attitudes positives au travail). Les épreuves à franchir pour être embauché sont très difficiles et seule une 